\title{
ОБ ОСОБЕННОСТЯХ ОПРЕДЕЛЕНИЯ СРЕДНЕСУТОЧНЫХ ВЕЛИЧИН АЛЬБЕДО В ВЫСОКИХ ШИРОТАХ
}

\section{Ю.А. Скляров, Н.В. Фомина, А.И. Котума, Н.В. Семенова}

Саратовский государственный университет, кафедра метеорологии и климатологии

E-mail: kafmeteo@sgu.ru,

Статья является продолжением опубликованной ранее работы и излагает особенности метода определения среднесуточного альбедо в высоких и околополярных широтах. Приводятся примеры расчетов.

Ключевые слова: среднесуточное альбедо, бин, широта, склонение Солнца, полярный день.

Concerning the Peculiarities in Determination of Daily Averaged Albedo in High Latitudes

\section{Yu.A. Sklyarov, N.V. Fomina, A.I. Kotuma, N.V. Semenova}

The paper is continuation of a work, published earlier and gives the details of the method of determination of daily averaged albedo values in high and nearpolar latitudes. The examples of calculations are given.

Key words: Daily averaged albedo, bin, latitude, declination Sun, polar day.

В работе [1] изложена методика определения среднесуточных величин альбедо с использованием моделей направленного альбедо США [1-3], показывающих зависимость величин альбедо для 12 выбранных моделей (сцен) от зенитного угла Солнца (ЗУС). Основная идея предложенного метода заключается в нахождении суточного осредненного фактора. Для учета зависимости альбедо любой модели от ЗУС небесная сфера над измеряемой площадкой разделена на бины по зенитному углу Солнца - от зенита до горизонта. При этом угловая ширина бинов по зенитному углу определяется так, что разность косинусов зенитного угла для верхней и нижней границ бина составляет 0,1 . Это дает 10 бинов, ширина которых соответственно уменьшается от зенита (бин № 1, интервал ЗУС составляет 0-25,84) до горизонта (бин № 10, интервал ЗУС 84,26-90,00). При этом величина альбедо в зависимости от ЗУС $\alpha\left(Z_{\odot}\right)$ в пределах бина принимается одинаковой и рассчитывается по среднему значению косинуса для данного бина. Например, расчеты альбедо для бина № 4 (косинусы верхней и нижней границ 0,7 и 0,6$)$ производится со значением косинуса 0,65 . В работе достаточно подробно описана структура американских моделей направленного альбедо и рассмотрен ход их использования для оценки среднесуточных величин альбедо. При измерениях уходящей коротковолновой радиации (УКР) со

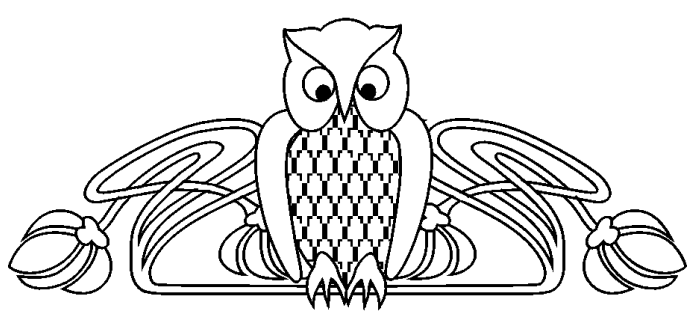

спутника, с учетом момента времени измерения, координат рассматриваемой площадки (центра ячейки выбранной сетки на верхней границе атмосферы) определяется конкретная величина альбедо такой площадки. Это - мгновенное значение альбедо, относящееся к конкретному значению ЗУС в момент измерения. Такие величины представляют интерес для ряда конкретных задач. Однако для использования в климатических исследованиях нужны сведения о среднесуточных (учет изменений, связанных с сильным суточным циклом) среднемесячных величинах альбедо. Именно эти величины позволяют рассчитывать энергетические потоки за сутки, месяц и далее среднегодовые. Очевидно, что мгновенная величина альбедо относится только к данному $Z_{\odot}$ в момент измерения. А в течение суток Солнце может проходить над данной площадкой целый ряд бинов, начиная с момента восхода (10-й бин) и до некоторого бина, в котором Солнце находится в момент верхней кульминации.

ЗУС легко определяется для этого момента по известной формуле для зенитного расстояния светила в верхней кульминации. Если $Z_{\odot}^{\text {B.к. }}-$ зенитный угол Солнца в этот момент, $\varphi$ - географическая широта рассматриваемой площадки, $\delta$ - склонение Солнца, то имеем

$$
Z_{\odot}^{\text {в.К. }}=\varphi-\delta,
$$

либо для низких широт при $\delta>\varphi$ :

$$
Z_{\odot}^{\text {B.K. }}=\delta-\varphi .
$$

У низких приэкваториальных широт небесный экватор пересекает меридиан недалеко от зенита. Суточные параллели Солнца могут проходить в пределах бина № 1. Тогда над площадкой в такой широтной зоне Солнце от восхода до кульминации будет проходить через все 10 бинов. А зависимость величины альбедо от ЗУС, согласно моделям, весьма значительна $[1,3]$. Например, для сцены «океан, ясно» (т.е. практически безоблачное небо над океаном) альбедо изменяется от 7,6\% (бин № 1, Солнце вблизи зенита) до 33,4\% (бин № 10)! Иными словами, альбедо одной и той же площадки меняется в течение дня (ночью не измеряется) в 4,4 раза! Даже для самой «гладкой» модели «сплошная облачность» изменение альбедо составляет 1,5 раза. Таким образом, если для расчетов отраженных или поглощенных потоков использовать мгновенное измеренное значение альбедо, то при оценке среднесуточных или среднемесячных величин легко впасть в ошибку. 
Например, мгновенное альбедо получено при высоком Солнце, пусть в бине № 2 (до $Z_{\odot} \approx 37^{\circ}$ ). Для нашего примера с моделью «океан, ясно» это будет $8,2 \%$. А Солнце пройдет от восхода все бины до № 2 (или даже № 1) включительно. Для бина № 10 альбедо площадки равно 33,4\%. Следовательно, за счет больших альбедо для низких высот Солнца среднесуточное альбедо будет иметь существенно большую величину, чем 8,2\% в момент измерения.

В работе [1] рассмотрена в деталях процедура получения среднесуточных величин альбедо для всех широт, при которых наблюдаются восходы и заходы Солнца. Тогда моменты восходов-заходов $t_{\text {в.3 }}^{\odot}$ рассчитываются по известной формуле

$$
\cos t_{\mathrm{B} .3}^{\odot}=-\operatorname{tg} \varphi \cdot \operatorname{tg} \delta,
$$

где $t_{\text {в.3 }}^{\odot}$ - часовой угол Солнца в моменты восхода, либо захода; $t_{\text {в.3 }}^{\odot}$ со знаком «+» дает часовой угол захода $t_{3}^{\odot}$, а $t_{\text {в.3 }}^{\odot}$ со знаком «-» соответствует часовому углу восхода (по правилам астрономии). Для получения среднесуточного значения альбедо надо найти средневзвешенную по инсоляции величину альбедо из всех его величин в бинах, которые проходит Солнце от восхода до кульминации (из-за симметрии суточной параллели Солнца по отношению к меридиану можно брать только ее половину). Очевидно, что весовые коэффициенты альбедо бинов будут пропорциональны времени нахождения Солнца в соответствующем бине. А сумма таких промежутков $\Delta t_{b}^{i}$ будет равна часовому углу восхода (или захода) Солнца. Найти $\Delta t_{b}^{i}$ можно, используя известное соотношение для расчетов часового угла Солнца:

$$
\cos t_{\odot}=\frac{\cos Z_{\odot}-\sin \varphi \sin \delta}{\cos \varphi \cos \delta} .
$$

Находя часовой угол $t_{b}^{i}$ для нижних границ бинов, получим $\Delta t_{b}^{i}=t_{b}^{i}-t_{b}^{i-1} ; t_{b}^{i}-$ часовой угол нижней границы $i$-го бина, а $t_{b}^{i-1}-$ то же для нижней границы $(i-1)$-го бина. Здесь нумерация бинов идет по нижним границам.

Это соответствует нумерации бинов, принятой в моделях. Например, бин №10 имеет нижнюю границу то же $i=10$. Солнце в нем находится от восхода до пересечения нижней границы $(i-1)$-го бина, т.е. 9-го и т.д. Определяя $t_{b}^{10}$ по формуле (1), a $t_{b}^{9}$ по формуле (2), получим промежуток времени нахождения Солнца в бине № $10: \Delta t_{b}^{10}=t_{b}^{10}-t_{b}^{9}$. Далее по формуле (2) аналогично определяются все остальные $\Delta t_{b}^{i}$, используя табличные значения зенитного угла $Z_{b}^{i}$ нижних границ бинов [1, 3]. Очевидно, что наиболее высоким бином, в котором может находиться Солнце для данной широты $\varphi$, склонения $\delta$, будет бин, где Солнце кульминирует. Из условия $Z_{\odot}^{\text {в.к. }}=\varphi-\delta$ либо $Z_{\odot}^{\text {в.к. }}=\delta-\varphi$ находится минимальное значение $Z^{\text {min }}$ Солнца в верхней кульминации. Ближайшая к нему граница бина с большим значением зенитного угла $Z_{b}^{\min }$ и есть нижняя граница бина. Промежуток времени пребывания в этом бине равен непосредственно часовому углу $t_{b}^{i}(\mathrm{~min})$, поскольку в момент верхней кульминации часовой угол Солнца равен нулю.

В работе [1] изложены подробности примененного метода. В частности, для учета конкретного значения альбедо в момент измерения $a\left(Z_{\odot}\right)$, а не среднего по бину, использованы нормированные величины модельных альбедо. Приведены примеры расчетов, карты глобальных распределений среднемесячных альбедо и др.

Мы уже отмечали, что предложенная методика расчетов среднесуточного значения альбедо охватывает все случаи сочетаний широт и склонений Солнца, при которых происходят его восходы и заходы. Однако в высоких и особенно приполярных широтах в различные времена года (влияние годовых изменений склонения Солнца в пределах от $-23^{\circ} 27^{\prime}$ до $+23^{\circ} 27^{\prime}$,т.е. подсолнечная точка перемещается по поверхности Земли почти на $47^{\circ}$ ) восходы и заходы отсутствуют. Очевидно, изложенная выше методика требует доработки.

Рассмотрим в общем виде особенности суточного движения Солнца на приполярной широте с тем, чтобы потом использовать их для решения задачи получения среднесуточных значений альбедо и обсуждения других вопросов.

Часть небесной сферы над площадкой с географической широтой $\varphi$, которая выбрана достаточно высокой, чтобы продемонстрировать особенности суточного движения Солнца в высоких широтах, изображена на рис. 1.

На рис. 1 показаны необходимые углы: широта $\varphi=\angle P O N$ и равные ей или зависящие от нее углы; склонение Солнца $\delta$; суточная параллель Солнца при склонении равном $\delta$. При таком склонении Солнце нигде не касается математического горизонта (круг $S E N$ на лицевой стороне), кульминирует в нижней кульминации над точкой севера $N$, что видно из рис. 1 . Это тот самый случай, когда Солнце не заходит, а кружит над горизонтом. После дня весеннего равноденствия $(\delta=0$, Солнце восходит в точке востока $E$, день равен ночи, так как зайдет оно в этот день в точке запада, т.е. половина суточной параллели над горизонтом и половина под горизонтом), продолжая движение по склонению к северу, Солнце через некоторое время в нижней кульминации коснется точки севера $N$. Точки восхода от точки $E$ движутся к точке $N$, день продолжает увеличиваться и в момент касания в нижней кульминации точки $N$ станет равным 24 часам (12 часов от нижней до верхней кульминации и столько же от верхней до нижней). С этого момента начинается полярный 


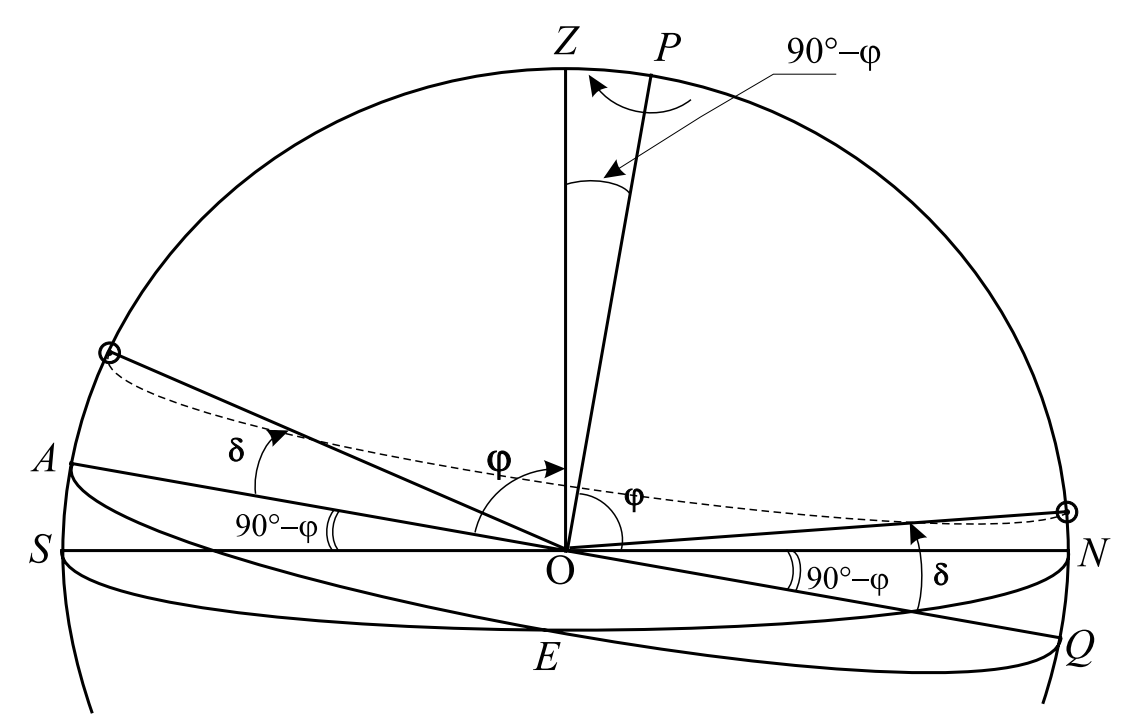

Рис. 1. К вопросу о полярном дне

день для широты $\varphi$, так как до 22 июня Солнце продолжает уходить к северу вплоть до склонения $+23^{\circ} 27^{\prime}$. Это день летнего солнцестояния. Затем явления повторяются в обратном порядке. В некоторый день Солнце снова коснется горизонта в нижней кульминации, полярный день закончится, начнется период восходов и заходов. Оценить продолжительность полярного дня несложно. Из рис. 1 видно, что при нижней кульминации Солнца в точке $N$ выполняется условие: $\delta_{\text {п }}=90^{\circ}-\varphi$. Например, пусть $\varphi=80^{\circ}$, тогда склонение $\delta_{\Pi}$, обеспечивающее минимальную продолжительность полярного дня в 24 часа, будет $\delta_{\Pi}=10^{\circ}$. По таблице склонений из астрономического ежегодника [4] находим, что этому склонению соответствует дата 16 апреля. Этому же склонению после дня солнцестояния соответствует дата 28 августа. Таким образом, для широты $80^{\circ}$ полярный день длится от 16 апреля по 28 августа. В этот период, более 4-х месяцев, Солнце на данной широте не заходит, методика расчетов среднесуточного альбедо [1] к данной ситуации неприменима, не действует формула (1), во всяком случае требуется коррекция.

Из проведенного обсуждения также следует, что условием для незаходящего Солнца будет следующее: $\delta \geq 90^{\circ}-\varphi$, а для заходящего и восходящего $-\delta<90^{\circ}-\varphi$. Так как предельное положительное склонение Солнца в день солнцестояния в нашу эпоху равно $+23^{\circ} 27^{\prime}$, то можно легко найти широту, начиная с которой появляются полярные дни. Очевидно, что это будет условие $90^{\circ}-\varphi_{\Pi}=23^{\circ} 27^{\prime}$, откуда $\varphi_{\Pi}=90^{\circ}-23^{\circ} 27^{\prime}=66^{\circ} 33^{\prime}$. Это и есть широта северного полярного круга $\varphi_{\text {п }}$. Именно на этой широте теоретически один раз в году, 22 июня, Солнце не заходит, а лишь касается горизонта в точке севера $N$. Таким образом, широта полярного круга является пределом, до которого в течение всего года применима методика, разработанная в [1], так как до этой широты всегда существуют восходы-заходы Солнца и действует формула (1).

Отметим еще одно обстоятельство. Рассмотрим ситуацию с верхними кульминациями Солнца (слева на рис. 1 , над точкой юга $S$ ). После 22 июня высота Солнца в верхней куль-

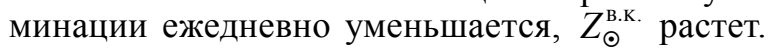
23 сентября Солнце кульминирует в точке $A$, на небесном экваторе, день равен ночи. Перейдя в Южное полушарие Солнце 22 декабря достигнет склонения $-23^{\circ} 27^{\prime}$. Но если широта равна $\varphi_{\text {п }}=66^{\circ} 33^{\prime}$, то склонение точки юга $\mathrm{S}$ с учетом знака (Южное полушарие), т.е. угол $\angle A O S$, тоже равно $\left(90^{\circ}-\varphi\right)=-23^{\circ} 27^{\prime}$. Таким образом Солнце в верхней кульминации попадает в точку юга снизу, из-под горизонта. Восхода не будет, вся суточная параллель Солнца пройдет под горизонтом. Следовательно, полярный круг - это географическая параллель, на которой Солнце один раз в год не заходит (22 июня) и один раз в год не восходит (22 декабря). Поскольку в Южном полушарии ситуация симметрична и обратна по отношению к временам года, то и Южный полярный круг играет точно такую же роль для южных широт.

Подведем некоторые итоги.

1. В зоне широт от южного до северного полярных кругов для любой широты и любого склонения Солнца (любого времени года) в каждый день года имеются восход и заход Солнца. Формула (1) действует, следовательно, действует и методика расчетов среднесуточного альбедо [1].

2. В приполярных широтах количество дней явлениями восход-заход сокращается на столько, на сколько появляется дней без заходов Солнца (полярный день). Это следует из выполнения условия $\delta>90^{\circ}-\varphi$. Пример расчета был приведен выше.

3. При приближении к полюсу убывает величина $90^{\circ}-\varphi$ и растет число дней полярного дня. Если $\varphi=90^{\circ}$, т.е. площадка находится на полюсе, то 
$90^{\circ}-\varphi=0$, т.е. полюс мира $P$ совпадает с зенитом, а небесный экватор $A E Q$ (см. рис. 1) совпадет с математическим горизонтом. При любых $\delta>0$ Солнце не будет заходить, а при $\delta<0$ будет кружить под горизонтом. Это тот случай, когда 21 марта Солнце пересечет экватор, поднимаясь в Северное полушарие. Это и будет день восхода. Через полгода Солнце уйдет в Южное полушарие. Один восход, один заход, полгода полярный день. После 21 марта Солнце, медленно поднимаясь почти по окружностям, последовательно проходит 10-й, 9-й... и пересекает нижнюю границу 6-го бина (ее зенитное расстояние $66^{\circ} 42^{\prime}$, а ЗУС 22 июня - 66 $33^{\prime}$ ). После этого бины проходятся Солнцем в обратном порядке. Нет надобности искать среднесуточное альбедо, просто нужно определить по $\delta_{\odot}$, в каком бине происходит суточное движение Солнца. Измеренное значение альбедо равно среднесуточному. А во всех других случаях, когда $\varphi>\varphi_{\Pi}$, но меньше $90^{\circ}$, усреднение по разработанной методике производится только для дней, когда $\delta<90^{\circ}-\varphi$, т.е. когда еще имеются восходы-заходы Солнца.

В случае незаходящего Солнца, при $\delta>90^{\circ}-\varphi$, усреднение можно выполнить следующим образом. Рассмотрим рис. 2. На нем изображена ситуация, когда $\delta>90^{\circ}-\varphi$, захода нет, Солнце в нижней кульминации проходит над точкой севера $(N)$. Изображен случай, когда Солнце кульминирует над нижней границей 9-го бина. Для оценки ЗУС воспользуемся формулой зенитного угла Солнца в нижней кульминации:

$$
Z_{\odot}^{\text {H.K. }}=180-\varphi-\delta .
$$

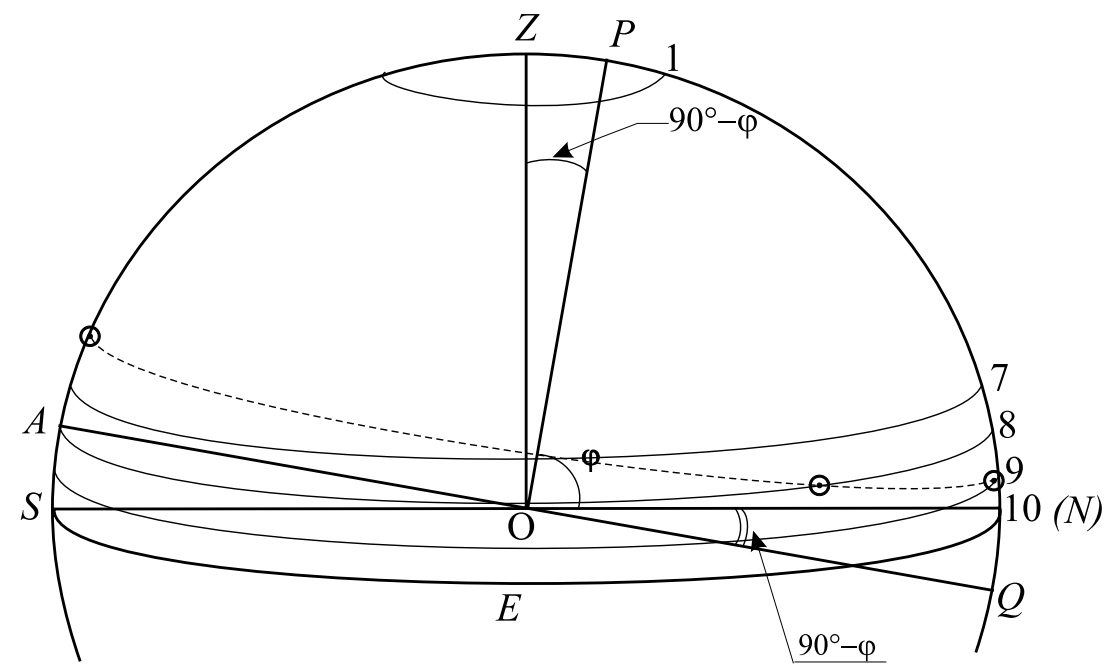

Рис. 2. К расчету среднесуточных альбедо в условиях полярного дня

Эта формула легко находится из рис. $1: Z_{\odot}^{\text {н.к. }}$ - это $\angle Z O_{\odot}$ (над точкой севера $\left.N\right)$. Он состоит из 2-х углов: $\angle Z O P=90^{\circ}-\varphi$ и $\angle P O_{\odot}=90^{\circ}-\delta$. Их сумма дает формулу (3). Находя по формуле (3) $Z_{\odot}^{\text {н.к. }}$ и сопоставляя его с табличными величинами нижних границ бинов, получим место Солнца в соответствующем бине в момент нижней кульминации (например, на рис. 2 зенитный угол нижней границы 9-го бина равен $84^{\circ}, 26[1,3]$, а ЗУС - меньше). Далее, вместо определения часового угла восхода Солнца сразу принимаем часовой угол в нижней кульминации 12 часов. Затем, подставляя в формулу (2) нижнюю границу 8-го бина, получим $t_{b}^{8}$ и время пребывания Солнца в бине 9: $\left(12\right.$ час $\left.-t_{b}^{8}\right)$ и далее, как и в предложенной методике [1]. Таким образом, в данном примере бин 10-й исключается из осреднения.

В заключение обсудим еще один вопрос. Расчет приходящей прямой солнечной радиации производится по формуле Миланковича для суточной суммы [5]. Переходя к среднесуточной плотности приходящего потока $\bar{E}\left[\mathrm{BT} \cdot \mathrm{M}^{-2}\right]$ запишем формулу в виде

$$
\bar{E}=\frac{E_{0}}{r^{2} \pi}\left(t_{s} \sin \varphi \sin \delta+\cos \varphi \cos \delta \sin t_{s}\right),
$$

где $E_{0}-$ солнечная постоянная; $r$ - расстояние Солнце-Земля в астрономических единицах; $t_{s}-$ часовой угол в радианах в момент восхода (захода) Солнца; $\varphi$ и $\delta$ - широта площадки и склонение Солнца соответственно.

В приполярных широтах формула меняет вид. Поскольку в случае полярного дня $t_{s}=\pi$, то $\sin$ $t_{s}=0$, и формула приобретает вид

$$
\bar{E}=\frac{E_{0}}{r^{2} \pi}(\sin \varphi \sin \delta) \text {. }
$$

Но конкретно на полюсе $\left(\varphi=90^{\circ}\right)$ угловая высота Солнца $h_{\odot}$ над горизонтом равна $\delta_{\odot}$. А $h_{\odot}=$ $=90^{\circ}-Z_{\odot}$, поэтому, с учетом того, что $\sin \varphi=1$ : 


$$
\bar{E}=\frac{E_{0}}{r^{2} \pi} \cos Z_{\odot} .
$$

Формула напоминает общую формулу падения радиации на поверхность. Она уменьшается пропорционально косинусу угла падения лучей с нормалью к поверхности. Все вышеизложенные соображения учтены при доработке программы расчетов.

\section{Библиографический список}

1. Скляров Ю.А., Бричков Ю.И., Фомина Н.В. и др. Определение среднесуточных величин альбедо с использовани- ем спутниковых направленных моделей // Исследование Земли из космоса. 2005. № 3. С. 13-21.

2. Suttles J.T., Green R.N., Minnis P. et al. Angular radiation models for Earth-atmosphere system. NASA Ref. Publ. 1184. Washington, 1998. $145 \mathrm{p}$.

3. Скляров Ю.А., Бричков Ю.И., Воробьев В.А. и др. Аэростатные измерения уходящей коротковолновой радиации // Исследование Земли из космоса. 1994. № 1. C. $11-20$.

4. Астрономический календарь на 1993 г. / Под ред. Д.Н. Пономарева. М., 1992. 288 с.

5. Миланкович М. Математическая климатология и астрономическая теория колебаний климата. М.; Л., 1939. $208 \mathrm{c}$. 